\title{
Management control in the automotive industry
}

\author{
Daria Battini · Nils Boysen
}

In spite of its long tradition, the automotive industry still produces a much sought-after consumer good and provides jobs for a large fraction of the workforce in many nations all around the world. Moreover, the automotive industry has given birth to many trends and problem solutions, some of them having reached other branches of industry only with a considerable delay. To name just a few recent ones there are:

1. the lean principle promoted by the famous Toyota Production System,

2. mass customization, which allows the customer to configure an individual car from a wide range of standardized options,

3. ergonomic operations, so that an efficient mass production can still be guaranteed in light of the aging workforce of many Western societies,

4. increasing ecological awareness initiating a fundamental redesign of the product, e.g., due to new drive concepts or car sharing,

5. quickly expanding, globalized supply chains to fulfill the growing demands in the Far East and South America, and

6. increasing uncertainty due to a growing product variety and decreasing product life cycles.

Thus, the automotive industry has been and still is a trendsetter for many other industries, so that the closer look of this special issue on new developments in scientific research and practical applications of management control in the automotive industry seems a worthwhile endeavor.

D. Battini

University of Padua, Padua, Italy

e-mail: daria.battini@unipd.it

N. Boysen (殴)

University of Jena, Jena, Germany

e-mail: nils.boysen@uni-jena.de 
This special issue presents five papers all dealing with a different subset of the aforementioned trends. The papers were selected on the basis of their fundamental ideas and concepts rather than the thoroughness of the techniques employed. It is our pleasure to note that all the papers are derived by practical experiences and real-world applications in the automotive field.

In the first paper, Dudenhöffer analyzes the problem of the electric vehicle acceptance in the automotive market by an experimental design where customers were able to test drive electric cars. For the experiment, eleven plug-in electric vehicles were tested by 232 customers. The author's analysis is based on an extension of the Technology Acceptance Model (TAM) and the results indicate that electric vehicles failed so far because funding programs by the government lacked support for test options for customers in order to experience this new drive concept.

The second paper by Häntsch and Huchzermeier deals with risk management in strategic planning of automotive production networks. The authors identify and assess significant risks to be carefully considered during the strategic planning process and especially address flexibility as a promising means to mitigate these risks. The applicability of their planning processes and risk measures are shown by a real-world case of a major German car manufacturer.

The third paper by Farnoush and Wiktorsson is dedicated to identifying the right production control mechanism for the divergent assembly structure of a power train manufacturer. In a large simulation experiment with real-world shop-floor data typical control mechanisms like POLCA and CONWIP are evaluated with regard to their resulting impact on the work-in-process and throughput. This way, some decision support is offered for the practitioner having to select the right control mechanism in the widely unexplored field of divergent assembly structures.

Two additional papers deal with the "part feeding problem" and the idea of applying decentralized storage areas on the shop floor (also denoted as "supermarkets") in order to supply car assembly with a wide variety of parts. Supermarkets are an emerging trend in in-house logistics. They enable a reliable and flexible small-lot delivery of parts in line with the basic Just-in-Time (JIT) philosophy.

The fourth paper, written by Faccio et al., proposes an integrated approach both for long- and short-term decision problems occurring when setting up the supermarket concept. A holistic planning approach is introduced in order to size and manage the tow train fleet delivering parts from the supermarket to final assembly. The proposed methodology is applied to a case study of an Italian car producer and the results highlight the dire need for integration of long- and short-term aspects in order to successfully solve the part feeding problem under investigation.

Finally, Battini et al. present a short survey paper on the supermarket concept. The basic setup in all its alternative configurations is described in detail and a literature survey on elementary decision problems and solution concepts is provided.

The guest editors wish to thank all the authors of this special issue for their interesting contributions. We would also like to thank the managing editor of JoMaC for the support and efficient collaboration, and all referees who have evaluated the papers in a very constructive spirit within the short stipulated time. 